\title{
Insegurança alimentar intrafamiliar e perfil de consumo de alimentos ${ }^{1}$
}

\author{
Intra-family food insecurity and \\ profile of food consumption
}

\author{
Giseli PANIGASSI ${ }^{2}$ \\ Ana Maria SEGALL-CORRÊA ${ }^{2}$ \\ Letícia MARIN-LEÓN² \\ Rafael PÉREZ-ESCAM ILLA ${ }^{3}$ \\ Lucia Kurdian MARANHA ${ }^{2}$ \\ Maria de Fátima Archanjo SAM PAIO²
}

\section{R E S U M O}

\section{Objetivo}

Descrever e avaliar o perfil de consumo diário de alimentos entre famílias em situação de insegurança alimentar.

\section{Métodos}

Inquérito populacional realizado em Campinas (SP), em 2003, com 456 famílias. Realizou-se levantamento do consumo diário de 14 grupos de alimentos, mediante informação de membro qualificado da família. Para categorização da insegurança alimentar utilizou-se uma escala dividida em 3 catego rias: 1) Segurança alimentar; 2) Insegurança alimentar leve; 3) Insegurança alimentar moderada ou grave.

\section{Resultados}

Foram detectadas diferenças significativas entre categorias de segurança alimentar e consumo alimentar. A proporção de famílias em situação de insegurança, cujo informante não consome diariamente leite e derivados, frutas, verduras/legumes, e carnes é significantemente maior do que aquelas em situação de segurança alimentar $(p<0,001)$. Nas famílias em segurança alimentar, o consumo de pelo menos uma fruta diariamente foi $73,7 \%$ e de derivados do leite $62,1 \%$. Essas proporções são $11,4 \%$ e 5,5\%, respectivamente, em famílias que experimentam insegurança alimentar moderada ou grave. Nestas últimas, a maioria consome diariamente apenas cereais, óleo, açúcar e feijão e gasta cerca de $68,0 \%$ da renda com despesas em alimentação. Existem

\footnotetext{
1 Projeto de Pesquisa "Validação de metodologia e instrumento de coleta de informação". Apoio: Fundação de Amparo à Pesquisa do Estado de São Paulo - Bolsa Professor Visitante: Dr. Rafael Pérez-Escamilla. Ministério da Saúde DECITUNESCO/FCM/Segurança al imentar Sul, Sudeste e Centro-Oeste - Contrato Funcamp: no SA- 9811/2003, MDS-Unesco/ FCM/Segurança alimentar Norte e Nordeste - Contrato: Processo no SA- 3382/2004.

2 Universidade Estadual de Campinas, Faculdade de Ciências Médicas, Departamento de Medicina Preventiva e Social. R. Tessália Vieira de Camargo, 126, Cidade U niversitária, 13081-970, Campinas, SP, Brasil. Correspondência para/Correspondence to: G. PANIGASSI. E-mail: <panigi@unicamp.br>.

3 University of Connecticut, Department of Nutritional Sciences. Storrs, CT, United States.
} 
diferenças significantes na freqüência das principais refeições diárias entre as categorias de segurança, sempre com menor freqüência entre os informantes das famílias em insegurança alimentar moderada ou grave.

\section{Conclusão}

Famílias em insegurança alimentar moderada ou grave apresentaram dieta monótona, basicamente composta por alimentos energéticos. A condição de acesso ao alimento entre famílias em segurança alimentar, entretanto, não garantiu a adequação qualitativa da dieta. Esses resultados trazem a necessidade de reforçar, nas políticas de segurança alimentar, ações educativas direcionadas à promoção de alimentação saudável.

Termos de indexação: Consumo alimentar. Fome. Inquéritos sobre dietas. Insegurança alimentar.

\section{A B S T R A C T}

\section{Objective}

Describe and evaluate daily food consumption among families experiencing food insecurity.

\section{Methods}

A population survey was conducted in Campinas, São Paulo, in 2003, with 456 families. One qualified member of each family was interviewed about their daily intake of 14 food groups. For the food insecurity classification, a scale divided into three categories was used: 1) Food security; 2) Mild food insecurity; 3 ) Moderate or Severe food insecurity.

\section{Results}

Significant differences were found among the categories of food insecurity with respect to food consumption. The proportion of respondents from families experiencing moderate or severe food insecurity or mild food insecurity that did not consume dairy products, fruits, vegetables, meats, on a daily basis is significantly higher than respondents from families who are food-secure $(p<0.001)$. Among food-secure families, $73.7 \%$ consumed at least one fruit per day and $62.1 \%$ consumed dairy products every day. These percentages were $11.4 \%$ and $5.5 \%$, respectively, in families with moderate or severe food insecurity. The majority of these families consumed only grains, oil, sugar, and dried beans on a daily basis, and they spend roughly $68 \%$ of their monthly income on food. There are significant differences in the frequency of the main daily meals among the food security categories, which is always less frequent among families with moderate or severe food insecurity.

\section{Conclusion}

Families experiencing moderate or severe food insecurity have a boring diet, composed basically of energetic foods. Even among food secure families, adequate diet quality was not always present. These results show that it is necessary to strengthen nutrition education efforts in public policies that aim to improve food security and promote healthy diets.

Indexing terms: Food consumption. Hunger. Diet surveys. Food insecurity.

\section{N T R O D U Ç Ã O}

A segurança alimentar intrafamiliar, tal como conceituada pelo Conselho Nacional de Segurança Alimentar, é alcançada quando todos os seus membros têm acesso, por meio socialmente aceitável, ao consumo de alimentos em quantidade suficiente e de qualidade adequada, podendo levar assim, cada um deles, uma vida produtiva e saudável ${ }^{1}$.

Pensada dessa forma, a segurança alimentar impõe compreensão abrangente do que venha a ser uma dieta adequada. Ela deve suprir as necessidades energéticas do indivíduo, ser nutricionalmente diversificada, respeitar a idade, as condições fisiológicas, a atividade física e, por fim, os hábitos culturais de cada um deles²,3.

Graus agudos de deficiência alimentar podem ser sinônimos de fome, mas nem sempre a carência alimentar expressa automaticamente tal fenômeno. A fome crônica, silenciosa, talvez seja a forma de carência mais difícil de ser identificada, mas nem por isso, menos relevante. Observa-se que, nessa situação, o indivíduo troca 
a qualidade dos alimentos e, também, da dieta, procurando otimizar seus recursos financeiros. Posteriormente, com pouco ou nenhum recurso financeiro, começa a diminuir a quantidade de alimentos disponível para consumo da família, prioritariamente dos adultos. São estratégias de enfrentamento das restrições orçamentárias familiares e de priorização do consumo alimentar de um membro da família eleito como mais vulnerável ou mais importante ${ }^{4-6}$. Essa fome insatisfeita, prolongada ou apenas parcialmente saciada, cria vulnerabilidades e muitas vezes traduz-se em doenças ${ }^{7}$. A dieta pobre em carboidratos complexos e rica em açúcares simples e gorduras está associada à obesidade e a outras doenças crônicas como diabetes melitus tipo 2 e hipertensão arterial, levando à perda da qualidade de vida e, conseqüentemente, a um maior risco de morbimortalidade, principalmente por doenças cardiovasculares ${ }^{8}$.

Considera-se necessário que os estudos sobre insegurança alimentar englobem tanto 0 problema da situação de insuficiência alimentar em grupos mais vulneráveis da população brasileira, quanto às características qualitativas da dieta, buscando identificar os seus desvios das recomendações nutricionais.

O objetivo deste estudo foi o de comparar o perfil de consumo de alimentos entre famílias em insegurança alimentar com o padrão daquelas que não vivenciam tal problema, diagnosticadas por meio de escala de insegurança alimentar familiar validada para a realidade brasileira Escala Brasileira de Insegurança Alimentar (EBIA $)^{9,10}$.

\section{M É TO D O S}

Realizou-se, entre os meses de julho e agosto de 2003, inquérito populacional na cidade de Campinas, com amostra representativa de diferentes níveis sociais, medidos pelo nível de escolaridade do chefe da família. Utilizou-se amostragem probabilística por conglomerados em dois estágios, sendo o setor censitário a unidade primária de amostragem e o domicílio a unidade de amostragem do segundo estágio. A amostra do estudo foi constituída pela população não institucionalizada, residente na zona urbana do M unicípio de Campinas.

Para o sorteio da amostra foi usado o cadastro do Inquérito de Saúde no Estado de São Paulo (ISA-SP) ${ }^{11}$, formado pela relação de todos os domicílios existentes em 30 setores censitários do município de Campinas. Para viabilizar as análises segundo subgrupos sociais, o ISA-SP organizou a totalidade de setores censitários de 1996 em três estratos de nível socioeconômico, definidos segundo os percentuais de chefes de família com nível universitário: menos de 25\% (baixo), de 25\% a $50 \%$ (médio) e mais de $50 \%$ (alto). A seguir sorteou 10 setores em cada estrato.

Os setores sorteados foram percorridos, no ano 2000, por pesquisadores de campo do ISA-SP'11, que fizeram o arrolamento de todos os domicílios existentes, tendo como base croquis com os contornos dos quarteirões, fornecidos pelo Instituto Brasileiro de Geografia e Estatística (IBGE).

A partir da relação de todos os endereços registrados por setores censitários, sortearam-se aleatoriamente 1.000 domicílios para o projeto de avaliação da insegurança alimentar na população urbana de Campinas, obedecendo a uma proporção de 400 nos estratos de baixa e média escolaridade e 200 no estrato de alta escolaridade. Os parâmetros utilizados para cálculo da amostra foram os seguintes: no estrato de baixa escolaridade $(n=322)$ prevalência de $16,0 \%$ (erro aceitável de $12-20 \%$ ) e intervalo de confiança IC $95 \%$, no de média escolaridade $(n=331)$ $\mathrm{P}=8,5 \%$ (erro aceitável 5,5-11,5\%) e IC95\% e no de alta escolaridade $(n=171) \mathrm{P}=2,2 \%$ (erro aceitável 0-4,4\% ) e IC 95\%.

Dos mil domicílios sorteados foi possível incluir no estudo 847. Essa perda de 15,3\%, dentro do previsto no planejamento que propunha entrevistar 824 famílias, deu-se, na sua maioria, por dificuldades de encontrar moradores no domicílio sorteado mesmo após revisitas aos finais de semana. Entre as 847 famílias, apenas 456 tinham, pelo menos, um morador com menos de 
18 anos de idade e foram estas famílias que se tornaram objeto desta análise, pois esta é a condição necessária para aplicação da escala EBIA completa, com 15 questões. As famílias que não possuíam um integrante com menos de 18 anos de idade responderam apenas 8 questões da EBIA e foram excluídas do presente estudo.

0 informante entrevistado era aquele que tinha conhecimento da dinâmica da alimentação da família e com idade maior ou igual a 18 anos.

Para medir o nível de segurança alimentar, foi utilizado um instrumento previamente validado em amostra intencional de populações urbanas de quatro cidades brasileiras ${ }^{10}$. São 15 questões relativas à segurança alimentar familiar percebida nos últimos 3 meses, cuja somatória de respostas positivas configuram um escore de segurança/ insegurança para cada uma das famílias.

Foram usadas três categorias de análise desse escore: 1) condição de segurança alimentar (SA): não ter respostas positivas; 2) condição de insegurança alimentar leve (IAL): de 1 a 5 respostas positivas; e 3) condição de insegurança alimentar moderada (6 a 10 respostas afirmativas) ou grave: de 11 a 15 respostas afirmativas. A opção de analisar em conjunto o nível moderado com o grave se deve ao número reduzido de famílias em algumas estratificações de variáveis na análise dos dados.

O consumo diário de 14 alimentos ou grupos de alimentos, que compõem o padrão usual da alimentação da população brasileira, foi medido pela referência do consumo do informante da família (sim/não), aqui considerado uma proxy do consumo familiar e, por isso mesmo, assumido como possível de ser cotejada com os diferentes níveis de segurança alimentar intrafamiliar. A análise da freqüência de realização diária das refeições principais - café da manhã, almoço e jantar - também foi baseada na referência do informante da família. Realizou-se uma análise comparativa do perfil de consumo de alimentos segundo nível de segurança/insegurança alimentar.

Para constatar a asso ciação entre variáveis categóricas, foi utilizado o teste Qui-quadrado, sendo considerada significante a associação com $p$-valor $\leq 0,05$.

$\mathrm{Na}$ análise dos gastos familiares com a alimentação e de níveis de segurança foi calculado o odds proporcional mediante regressão logística politômica univariada, pois a variável resposta tem três categorias e segue uma ordem natural. Foi realizada a razão de prevalências de consumo de alimentos entre as categorias de SA e insegurança alimentar moderada ou grave (IAMG).

O banco de dados foi organizado no programa Statistical Packadge Social of Sciences (SPSS) para W indows (versão 6.0) e analisado no Programa Stata (versão 7.0), o qual permitiu o cálculo das estimativas das prevalências populacionais considerando as ponderações do desenho amostral. Os detalhes desses procedimentos foram relatados em outra publicação ${ }^{9}$.

Este projeto foi aprovado pela Comissão de Ética da Faculdade de Ciências M édicas, Universidade Estadual de Campinas, sob o parecer no 355/2003.

\section{RESULTA DOS}

Em Campinas, 39,5\% das famílias estudadas, com menores de 18 anos de idade, está em situação de segurança alimentar (SA). Entre aquelas que experimentam insegurança alimentar, 40,1\% referem preocupação de ficar sem alimento para garantir o futuro imediato, ou têm comprometimento qualitativo da dieta por limitação financeira, indicando insegurança alimentar leve, sem restrição quantitativa de alimentos (IAL). Outros $13,8 \%$ e $6,6 \%$ têm, respectivamente, insegurança alimentar moderada e insegurança alimentar grave, situações em que há restrição quantitativa na dieta entre adultos e/ou entre crianças.

A grande maioria dos entrevistados relatou fazer as três refeições principais todos os dias: $90,6 \%$ o café da manhã, 96,1\% o almoço e 92,9\% o jantar; porém há distinção quando são consideradas essas freqüências nas diferentes 
categorias de segurança/insegurança. Tanto as famílias em IAM G quanto as em IAL diferem significantemente das em SA em relação à ausência de realização diária de alguma das três principais refeições, sendo maiores as freqüências nas famílias em IAMG (Tabela 1).

A média de renda familiar mensal e a média de despesas efetuadas com alimentação, demonstradas na Tabela 2, têm grande variabilidade e são maiores no grupo de famílias em SA, sendo a proporção da renda gasta com a alimentação significantemente maior $(p<0,05)$ no grupo com IAMG. Nestas últimas famílias, mais de dois terços dos rendimentos mensais são destinados à alimentação, restando apenas 32\% para cobrir os gastos com as demais prioridades familiares.

Ao analisar os gastos familiares mensais com alimentação como variável independente e a categ oria de segurança alimentar, como dependente, na regressão logística politômica univariada, observa-se um aumento na razão de chance de a família estar em condição de IAM G de 46,4\% (IC 1,30-1,65 e $p=0,0001$ ) para cada 100 reais mensais a menos gastos com alimentação, e de

Tabela 1. Proporção de informantes das famílias que não realizam as refeições diariamente por categoria de segurança alimentar. Campinas (SP), 2003.

\begin{tabular}{|c|c|c|c|c|}
\hline Refeição & SA & $\begin{array}{l}\text { IAL } \\
\%\end{array}$ & IAMG & p-valor* \\
\hline Café da manhã & 4,6 & 9,3 & 19,6 & 0,0004 \\
\hline Almoço & 1,9 & 2,3 & 11,2 & 0,0003 \\
\hline Jantar & 3,5 & 7,7 & 13,2 & 0,0123 \\
\hline
\end{tabular}

*Teste Qui-quadrado; SA: segurança alimentar; IAL: insegurança alimentar leve; IAMG: insegurança alimentar moderada ou grave. estar em IAL de 7,5\% (IC 1,03-1,12 e $p=0,0004$ ), comparativamente às famílias em SA.

Ao comparar o consumo alimentar entre as três categorias de segurança/insegurança (Tabela 3), aparece uma forte e significante tendência decrescente de consumo de carne, leite, derivados do leite, frutas e verduras/legumes. Por outro lado, há maior consumo de todos esses grupos entre os informantes das famílias em SA, sendo menor entre aquelas em IAL e diminuindo mais entre as que apresentaram IAM G. Doces e refrigerantes também apresentaram tendência decrescente e significante, sendo menos consumidos pelas famílias em IAL e IAM G. Separando os alimentos por grupos, observa-se que os alimentos construtores, portanto, fontes de proteínas (carne, leite e derivados do leite) são consumidos por menos da metade das famílias em IAMG e, no caso dos derivados do leite, a proporção de famílias em SA que os consomem é 11 vezes maior. Diferenças semelhantes são encontradas, entre esses dois níveis de segurança alimentar, na análise do consumo de alimentos reguladores (frutas, verduras e legumes), ricos em fibras, vitaminas e minerais. M esmo entre os membros de famílias em IAL sem restrição quantitativa, o consumo destes últimos alimentos é baixo: $35 \%$ dos entrevistados não incluem diariamente legumes e verduras em suas dietas e $56 \%$ não consomem frutas na mesma freqüência.

A grande maioria dos membros de famílias em IAM G refere o consumo diário, além do feijão, de alimentos densamente energéticos como os cereais, óleo e açúcar. Esse fato também é constatado nas famílias em IAL, porém, nestas, mais de $70 \%$ incluem leite na sua dieta diária.

Tabela 2. Médias da renda e da despesa familiar mensal com alimentação por categoria de segurança/insegurança alimentar. Campinas (SP), 2003.

\begin{tabular}{|c|c|c|c|c|c|c|}
\hline \multirow{2}{*}{ Classificação } & \multicolumn{2}{|c|}{ SA } & \multicolumn{2}{|c|}{ IAL } & \multicolumn{2}{|c|}{ IAMG } \\
\hline & M & DP & M & DP & M & DP \\
\hline Renda familiar mensal em Reais & 2187,59 & 1551,66 & 1198,22 & 1150,99 & 543,80 & 471,25 \\
\hline Despesa familiar mensal com alimentação em Reais & 886,73 & 649,61 & 618,43 & 522,73 & 370,92 & 304,96 \\
\hline Renda gasta com alimentação (\%) & \multicolumn{2}{|c|}{40,5} & \multicolumn{2}{|c|}{51,6} & \multicolumn{2}{|c|}{68,2} \\
\hline
\end{tabular}

SA: segurança alimentar; IAL: insegurança alimentar leve; IAM G: insegurança alimentar moderada ou grave. M: Média; DP: desvio-padrão. 
Tabela 3. Características de consumo diário de alimentos pelo informante da família, apresentadas segundo categorias de segurança alimentar, insegurança alimentar leve e insegurança alimentar moderada ou grave. Campinas (SP), 2003.

\begin{tabular}{|c|c|c|c|c|c|}
\hline Alimento & SA & $\begin{array}{l}\text { IAL } \\
-\%\end{array}$ & IAMG & $\begin{array}{l}\text { Razão de prevalência } \\
\text { (SA/IAM G) }\end{array}$ & p-valor* \\
\hline Óleo & 96,3 & 98,4 & 88,1 & 1,1 & 0,0002 \\
\hline Açúcar & 84,1 & 92,6 & 87,1 & 1,0 & 0,0407 \\
\hline Refrigerante & 44,5 & 38,8 & 11,5 & 3,9 & 0,0001 \\
\hline Doces & 38,6 & 19,8 & 5,3 & 7,3 & 0,0001 \\
\hline Carne & 85,1 & 65,9 & 32,3 & 2,6 & 0,0001 \\
\hline Leite & 83,0 & 74,1 & 46,2 & 1,8 & 0,0001 \\
\hline Derivados do leite & 62,1 & 24,3 & 5,5 & 11,3 & 0,0001 \\
\hline Ovo & 23,5 & 28,5 & 28,1 & 0,8 & 0,5035 \\
\hline Embutidos & 16,1 & 15,5 & 11,8 & 1,4 & 0,6243 \\
\hline Feijão & 90,4 & 91,8 & 85,5 & 1,1 & 0,2537 \\
\hline Verduras e legumes & 91,7 & 65,6 & 42,0 & 2,2 & 0,0001 \\
\hline Frutas & 73,7 & 43,8 & 11,4 & 6,5 & 0,0001 \\
\hline Cereais & 100 & 98,8 & 94,0 & 1,1 & 0,0173 \\
\hline Raízes & 44,2 & 33,4 & 26,1 & 1,7 & 0,0075 \\
\hline
\end{tabular}

*Teste do Qui-quadrado; SA: segurança alimentar; IAL: insegurança alimentar leve; IAM G: insegurança alimentar moderada ou grave.

Os entrevistados, membros de famílias classificadas em SA, relatam dieta mais diversificada. A maioria consome diariamente alimentos construtores, energéticos e reguladores, mas, mesmo assim, há uma parcela considerável que não possui o hábito de consumir frutas e derivados do leite diariamente.

A análise da associação entre níveis de segurança e consumo de feijão, ovos e embutidos mostra que esses alimentos não são sensíveis $(p>0,05)$ à condição de insegurança alimentar (Tabela 3).

\section{I S C U S SÃ 0}

A opção por estudar a qualidade da alimentação a partir do conhecimento dos alimentos que entram na composição da dieta diária, não permite uma inferência sobre a adequação quantitativa de energia, de macro e micronutrientes, porém atende às necessidades de avaliação da diversidade da dieta. Esta limitação é compensada pelo conhecimento já disponível de que dieta com maior diversificação dos alimentos, além de estar associada à adequação de nutrientes, também se associa à sua adequação energética ${ }^{2}$. Outro fator que deve ser considerado é a análise do consumo de alimentos, que, na verdade, é o relato do consumo do informante usado como variável proxy do consumo familiar e que, neste estudo, mostrou-se associado, na maioria dos alimentos, às categorias de segurança alimentar.

A situação de insegurança alimentar familiar observada em Campinas é intermediária entre aquela encontrada em Java, durante uma crise econômica da Indonésia em 1998, e a observada nos EUA em 1995. Na Indonésia, encontraram-se $94,2 \%$ de famílias em insegurança alimentar e $32,0 \%$ das famílias em situação de insegurança grave $^{12}$. No inquérito populacional americano, observou-se prevalência de insegurança alimentar em $11,9 \%$ das famílias investigadas, sendo que $4,1 \%$ delas foram diagnosticadas em situação de insegurança e fome ${ }^{13}$.

Ao comparar o resultado da pesquisa de Campinas com dados brasileiros, coletados por meio da EBIA, na Pesquisa Nacional por Amostra de Domicílios de 2004 (PNAD) ${ }^{14}$, verifica-se que 0 percentual de famílias em situação de insegurança leve é bem maior no município $(40,1 \%)$ do que na região Sudeste $(19,7 \%$ ), ou mesmo no Brasil $(20,3 \%$ ). Porém, analisando a insegurança alimentar moderada ou grave (IAMG) constata-se uma proximidade do percentual do País em geral 
(21,6\% ) com o de Campinas (20,4\% ). Já a região Sudeste $(13,5 \%$ ) permanece com percentual abaixo do valor encontrado no município estudado.

As investigações citadas, à semelhança da pesquisa realizada em Campinas, utilizaram instrumento para medida da segurança alimentar baseado na escala da fome de Radimer/Cornell15,16, originada de exaustivos estudos qualitativos, bem como na escala do Projeto de Identificação da Fome na Infância (CHIP) ${ }^{17}$. Tem sido demonstradas, em diferentes contextos sociais e culturais, a consistência e a adequação do uso dessa escala, como uma possibilidade de medida direta da segurança/insegurança alimentar, no âmbito da família. Tal insegurança é percebida em seus vários níveis, desde a preocupação de que o alimento venha a acabar antes que haja dinheiro para comprar mais, o que configura insegurança psicológica, passando, em seguida, pela insegurança relativa ao comprometimento da qualidade da dieta, porém ainda sem restrição quantitativa, até chegar ao ponto mais severo, que é a insegurança quantitativa, situação em que a família passa por períodos concretos de restrição na disponibilidade de alimentos para seus membros ${ }^{15}$.

Em Campinas, os gastos com aquisição de alimentos resultaram em um peso muito distinto na composição das despesas das famílias nas três categorias de análise, porém, em todas elas, representam uma parcela significativa do orçamento. Este comprometimento chega a 68,2\% do total da renda familiar no grupo em IAMG, muito próximo ao observado por estudo clássico de Josué de Castro com 500 famílias de trabalhadores do Recife, há mais de 7 décadas. Nessas famílias, cerca de $70,0 \%$ da renda salarial era consumida com sua alimentação ${ }^{18}$. Na Pesquisa de Orçamento Familiar de 2002/2003 (POF $2002 / 2003)$, esses percentuais variaram entre $32,7 \%$ da renda dasfamílias mais pobres até $9,0 \%$ da renda das mais ricas ${ }^{19}$. As diferenças encontradas entre a POF 2002/2003 podem ser atribuídas tanto à distinção do instrumento de coleta de informações das duas pesquisas, quanto à forma de análise, pois a estratificação da POF é feita por nível de renda e neste trabalho a análise foi realizada por categorias de segurança/insegurança alimentar.

O problema enfrentado por estas famílias em IAM G, resultado do alto custo da alimentação relativamente às disponibilidades orçamentárias pode, além de comprometer a própria alimentação, comprometer o acesso a outros bens e serviços essenciais a uma vida digna e de qualidade.

O inquérito de Campinas permitiu, ainda, a observação do evento "fome silenciosa" ou, como nos relatos de Valente ${ }^{3}$, "fome do dia". Comparando os grupos de segurança/insegurança alimentar deste estudo, percebeu-se que existem diferenças entre eles quanto à oportunidade de realizarem as três principais refeições do dia. Informantes de famílias em IAMG relatam com mais freqüência deixar de fazer, pelo menos, uma das refeições, como café da manhã, almoço ou jantar, gerando períodos diários longos sem alimentação. Nesta situação, crianças ou adultos ficam sem se alimentar durante um dia ou um período deste.

Observou-se, ainda, que mesmo as famílias em SA, portanto com garantia de acesso aos alimentos, apresentaram inadequações qualitativas na dieta tomando o entrevistado como referência; $26,3 \%$ não comem pelo menos uma fruta diariamente, contrapondo-se à estratégia de dieta saudável adotada pela Organização Mundial de Saúde $^{8}$. Cabe destacar o fato que 1 de cada 4 pessoas em SA não come pelo menos uma fruta por dia. De acordo com o Guia Alimentar para a População Brasileira, elaborado pelo M inistério da Saúde especificamente para essa população, e que visa contribuir para a melhoria dos perfis nutricional e epidemiológico por meio da alimentação saudável, a recomendação nutricional é de 3 porções diárias ${ }^{20}$. Surpreende ainda mais ao considerar os consumos elevados de refrigerantes $(44,5 \%)$ e doces $(38,6 \%)$, em substituição ao suco de frutas e à fruta como sobremesa na refeição brasileira tradicional, desaconselhados devido à presença do açúcar simples em grande quantidade. 
M onteiro et al. ${ }^{21}$, comparando os inquéritos realizados em áreas metropolitanas do Brasil entre os anos de 1988 e 1996, concluem que existe uma mudança nos padrões de consumo alimentar da população, caracterizada, principalmente, pela redução de carboidratos complexos e fibras e pela maior ingestão de açúcar simples.

A associação aqui encontrada, entre o baixo consumo de frutas, legumes e verduras e a insegurança alimentar, é consistente com os resultados de pesquisa realizada com adolescentes de Minneapolis, EUA ${ }^{22}$. No Brasil, essas disparidades na qualidade da alimentação de sua população já foram referidas, tomando a renda como fator de comparação ${ }^{19}$. Nos estratos superiores de renda mensal, o consumo médio de frutas chegou a ser quase 0 dobro do observado nos estratos inferiores, do mesmo modo que é diferente o perfil de consumo de legumes e verduras entre esses estratos ${ }^{23,24}$.

Barretto \& Cyrillo ${ }^{24}$, examinando a estrutura do consumo alimentar entre os anos de 1990 e 1996, verificaram que houve uma tendência decrescente dos gastos com alimentos in natura em todas as classes de renda ao longo desses anos. Os legumes, as frutas e as verduras, por suas propriedades decorrentes do seu conteúdo em fibras, minerais e vitaminas, são agentes importantes na prevenção das doenças crônicas não transmissíveis ${ }^{8,20}$. Portanto, a diminuição desses alimentos nas dietas, conforme descrito, representaria um risco potencial de maior freqüência dessas morbidades ${ }^{25}$.

À já evidente e bem explorada relação entre pobreza e insegurança alimentar, junta-se, atualmente, a preocupação, derivada de estudos mais recentes, sobre a relação entre insegurança alimentar e obesidade. No Brasil, vários estudos têm demonstrado a relação entre obesidade e vulnerabilidade socioeconômica ${ }^{26-28}$. Drewnowski $\&$ Specter ${ }^{29}$ sugerem que esse tipo de associação pode ser explicado pela correlação inversa existente entre o custo do alimento e sua densidade energética. Isto é, quanto mais denso em energia é o alimento, mais baixo é o seu custo, tornando- se assim, estes alimentos uma opção para os mais pobres.

A elevada proporção de famílias em IAM G que não consomem frutas nem verduras $(88,6 \%$ e $58,0 \%$, respectivamente) leva a supor que elas restrinjam sua dieta a alimentos de alto valor energético, em geral de menor custo, podendo este fenômeno explicar o agravamento da obesidade e suas co-morbidades, neste grupo populacional.

Sugere-se, ainda, que há necessidade de políticas públicas efetivas para apoiar as famílias na sua capacidade de elaborar estratégias para o enfrentamento do impacto das adversidades econômicas sobre a qualidade de suas dietas.

\section{O N C L U S Ã O}

O estudo apresentado mostra outro enfoque da iniqüidade social do Brasil, apontada aqui por um indicador de medida direta da insegurança alimentar e da fome. Em um dos mais prósperos municípios do País, a insegurança alimentar compromete a qualidade de vida de mais da metade de sua população e a insegurança com restrição no consumo quantitativo de alimentos, portanto com presença de fome entre adultos e/ou crianças (IAMG), afeta mais de 19 mil famílias em Campinas.

O custo da alimentação para grande parte das famílias, uma vez que isso absorve parcela significativa de suas rendas, é muito alto. Existe uma relação inversa entre os níveis de segurança/ insegurança alimentar e o percentual de comprometimento dos orçamentos das famílias.

A freqüência das refeições principais, ao longo do dia, diminui com o agravamento da insegurança alimentar. As famílias em insegurança alimentar com restrição quantitativa de alimentos apresentam uma dieta monótona, composta principalmente por alimentos de maior densidade energética. 0 acesso ao alimento, que está 
garantido para cerca de metade das famílias, em grande parte delas não Ihes assegura uma dieta saudável, em face à exclusão da dieta de frutas e verduras e ao hábito diário de consumir refrigerantes.

Estes resultados trazem a necessidade de reforçar, dentro das políticas de segurança alimentar e combate à fome, entre outras, ações educativas visando à promoção de alimentação balanceada e saudável para toda a população, independentemente de sua condição de acesso aos alimentos.

\section{A GRADECIMENTOS}

Organização Pan-Americana da Saúde, Ministério da Saúde, Ministério do Desenvolvimento Social e Combate à Fome e Fundação de Amparo à Pesquisa do Estado de São Paulo.

\section{COLA BORADORES}

G. PANIGASSI realizou levantamento bibliográfico, preparação do banco de dados, análise dos dados e redação do artigo. A.M. SEGALL-CORRÊA e L. M ARÍN-LEÓN orientaram a análise dos dados e a redação do artigo. R. PÉREZ-ESCAM ILLA, M.F.A. SAM PAIO e L.K. MARANHA realizaram contribuições à versão final do artigo.

\section{REFERÊ N CIAS}

1. Brasil. Conselho Nacional de Segurança Alimentar. Relatório final da II Conferência Nacional de Segurança Alimentar e Nutricional; 2004 mar. 17-20; Olinda.

2. Ruel MT. Is dietary diversity an indicator of food security or dietary quality? A review of measurement issues and research needs. Food Nutr Bull. 2003; 242(2):231-2.

3. Valente FLS. Do combate à fome à segurança alimentar e nutricional: 0 direito à alimentação adequada. Rev Nutr. 1997; 10(1):20-36.

4. Cristofar SP, Basiotis PP. Dietary intakes and selected characteristics of women ages 19-50 years and their children ages 1-5 years by reported perception of food insufficiency. J Nutr Educ. 1992; 24:53-8.
5. Rose D, Oliveira V. Nutrient intakes of individuals from food insufficient households in the United States. Am J Public Health. 1997; 87(12):1956-61.

6. Rose D. Economic determinants and dietary consequences of food insecurity in the United States. J Nutr. 1999; 129(Suppl):517-20.

7. Batista-Filho $M$, Rissin $A$. A transição nutricional no Brasil: tendências regionais e temporais. Cad Saúde Pública. 2003; 19(Suppl 1):181-91.

8. World Health Organization. Global strategy on diet, physical activity and health. Fifty-seventh world health assembly. [WHA57.17]. May 22; 2004.

9. Pérez-Escamilla R, Segall-Corrêa AM, Maranha LK, Sampaio MFA, Marín-León L, Panigassi G. An adapted version of the U.S. Department of Agriculture Food Insecurity Module is a valid tool for assessing household food insecurity in Campinas, Brazil. J Nutr. 2004; 134(8):1923-8.

10. Segall-Corrêa AM, Pérez-Escamilla R, Maranha LK, Sampaio MFA, Marin-Leon L, Panigassi G, et al. Acompanhamento e avaliação da segurança alimentar de famílias brasileiras: validação de metodologia e de instrumento de coleta de informação. Relatório técnico. M inistério da Saúde; mar 2004 [acesso 2004 dez 16]. Disponível em: <http:/ /www.opas.org.br/sistema/arquivos/vru_unic.pdf >.

11. César CLG, Carandiva L, Alves M CGP, Barros MBA, Goldbaum M. Saúde e condição de vida em São Paulo: inquérito multicêntrico de saúde no Estado de São Paulo-ISA-SP. São Paulo: USP; 2005.

12. Studdert LJ, Frongillo EA, Valo is P. Household food insecurity was prevalent in Java during Indonesia's economic crisis. J Nutr. 2003; 131(10):2685-891.

13. Bickel G, Carlson S, Nord M. Household food security in the United States, 1995-1998: Advance report. USDA, Food and Nutrition Service. Report 1999. Available from: <WWW.fns.gov/oane/menu/ published/foodsecurity/foodsec98.pdf >.

14. Instituto Brasileiro de Geografia e Estatística. Pesquisa nacional por amostra de domicílios: segurança alimentar 2004. Rio de Janeiro; 2006.

15. Radimer KL, Olson CM, Greene JC, Campbell CC, Habicht JP. Understanding hunger and developing items to assess it in women and children. J Nutr. 1992; 24(Suppl):36-44.

16. Radimer KL. Measurement of household food security in the USA and other industrialized countries. Public Health Nutr. 2002; 5(6A):859-64.

17. Wehler CA, Scott RI, Anderson JJ. The Community childhood identification project: a model of domestic hunger-demonstration project in Seattle, Washington. J Nutr. 1992; 24(Suppl):29-35. 
18. Castro J. Condições de vida das classes operárias do Recife. Recife: Imprensa Industrial; 1932.

19. Instituto Brasileiro de Geografia e Estatística. Coordenação de Índices de Preços. Pesquisa de orçamentos familiares 2002-2003 [acesso em 2004 dez 12]. Disponível em: <http://www.ibge. gov.br/home/estatistica/populacao/condicao devida /pof/2002/tab112.pdf>.

20. Brasil. M inistério da Saúde. Secretaria de Atenção à Saúde. Coordenação-Geral da Política de Alimentação e Nutrição. Guia alimentar para a população brasileira. Brasília: Ministério da Saúde; 2006.

21. Monteiro CA, Mondini L, Costa RBL. Mudanças na composição e adequação nutricional da dieta familiar nas áreas metropolitanas do Brasil entre 1988 e 1996. In: Monteiro CA, organizador. Velhos e novos males da saúde no Brasil: a evolução do país e de suas doenças. São Paulo: Hucitec; 2000.

22. Neumark-Sztainer D, Wall M, Perry C, Story $M$. Correlates of fruit and vegetable intake among adolescents findings from Project EAT. Prev Med. 2003; 37(3):198-208.

23. Maluf RS. Consumo de alimentos no Brasil: traços gerais e ações públicas locais de segurança alimentar. Pólis papers 2000; 6 [acesso em 2004 dez 12]. Disponível em: <http://www.polis.org.br/ publicacoes/papers/20006.html>.
24. Barretto SAJ, Cyrillo DC. Análise da composição dos gastos com alimentação no Município de São Paulo (Brasil) na década de 1990. Rev Saúde Pública. 2001; 35(1):52-9.

25. Brasil. Ministério da Saúde. A promoção da alimentação saudável como instrumento de prevenção e combate ao sobrepeso e obesidade. Documento Técnico. Brasília; 2004.

26. Monteiro CA, Conde WL, Castro IRR. A tendência cambiante da relação entre escolaridade e risco de obesidade no Brasil (1975-1997). Cad Saúde Pública. 2003; 19(Suppl.1):67-75.

27. Gigante DP, Dias-da-Costa JS, Olinto MTA, Menezes AMB, Macedo S. Obesidade da população adulta de Pelotas, Rio Grande do Sul, Brasil e associação com nível sócio-econômico. Cad Saúde Pública. 2006; 22(9):1873-9.

28. M arinho SP, Martins IS, Perestrelo JPP, Oliveira DC. Obesidade em adultos de segmentos pauperizados da sociedade. Rev Nutr. 2003; 16(2):195-201.

29. Drewnowski A, Specter SE. Poverty and obesity: the role of energy density and energy costs. Am J Clin Nutr. 2004; 79(1):6-16.

Recebido em: 6/3/2007

Versão final reapresentada em: 12/5/2008 Aprovado em: 16/5/2008 